\title{
In-situ strain measurements in composites during fatigue testing using optical fibre Bragg gratings and a portable CCD detection system
}

\author{
T. Liu, G. F. Fernando ${ }^{\#}$, Y. J. Rao ${ }^{\dagger}$, D. A. Jackson ${ }^{\dagger}$, L. Zhang ${ }^{\ddagger}$ and I. Bennion ${ }^{\ddagger}$ \\ Department of Materials Engineering, \\ Brunel University, Uxbridge, Middlesex, UB8 3PH. UK. \\ $\dagger$ Applied Optics Group, Physics Laboratory, University of Kent at \\ Canterbury, Kent CT2 7NR. UK. \\ $\ddagger$ Photonics Research Centre, Department of Electronic Engineering, \\ Aston University, Birmingham B4 7ET. UK. \\ \# To whom correspondence should be addressed.
}

\begin{abstract}
An optical fibre Bragg grating (FBG) sensor was surface mounted onto a carbon fibre reinforced epoxy composite and subjected to static and dynamic loading. The FBG sensor was found to operate satisfactorily up to 700,000 cycles when the fatigue test was terminated. The fatigue test was conducted at a peak stress of 210 $\mathrm{MPa}$ and a stress ratio of -0.5 . The FBG sensor was also found to operate satisfactorily over the cyclic loading frequency range of $0.1-6 \mathrm{~Hz}$ used in this study. The feasibility of using the sensor system for monitoring the stiffness of the composite during the fatigue test was demonstrated. The signal processing for the sensor system was based on a CCD spectrometer. The sensitivity of the static strain measurements was found to be approximately $80 \mu \varepsilon$. A broadening of the FBG reflective spectrum was seen to develop as a function of fatigue cycles, and this phenomenon may be due to the delamination of the sensor from the surface of the composite.
\end{abstract}

keywords: Smart structures, Optical Fiber sensors, Fibre Bragg grating, Strain, Fatigue damage, CCD, Fiber composites

\section{INTRODUCTION}

Significant progress has been made in recent years in the deployment of optical fibre-based sensor systems for strain monitoring in advanced fibre reinforced composites (AFRC). The primary driving force for this interest in strain monitoring is because this data can provide information on the integrity of the AFRC in question. In other words, if the AFRC sustains any damage (fibre fracture, delaminations, longitudinal splitting, matrix cracking or plasticization of the matrix by moisture etc.) the stiffness of the AFRC will be reduced ${ }^{[1]}$. Obviously, in areas where AFRCs are used for load-bearing applications, it is essential that the strength and stiffness of the AFRC are not degraded during service. Examples of typical events which can lead to structural damage in AFRC include, static and dynamic loading above a specified threshold, impact loading, cyclic temperature, sub-ambient and high temperature excursions, ingress of fluids which can degrade the matrix and/or the reinforcing fibres. Materials related parameters which can influence the nature of damage development in AFRCs include, the nature of the reinforcing fibre and volume fraction, void content, the type of matrix, the stacking sequence used in the construction of the composite, residual stresses, the nature of the interfacial bond strength between the matrix and the reinforcing fibres and edge effects.

Examples of optical fibre-based sensor systems which have been used for strain monitoring in AFRCs ${ }^{[2]}$ include intrinsic and extrinsic Fabry-Perot type sensors ${ }^{[3,4]}$, intensity-based sensors ${ }^{[5]}$, polarization-based sensors ${ }^{[6]}$ and optical fibre-based Bragg grating sensors ${ }^{[7,8]}$. From a materials viewpoint, the optical fibre Bragg sensors are ideally suited for deployment in AFRC structures because: (a) the FBG sensor is based on optical wavelength shift and hence is intrinsically immune to intensity drifts; (b) they can be readily multiplexed ${ }^{[9]}$; (c) compared with the Fabry-Perot sensor designs, the Bragg sensor construction is simpler; (d) the overall diameter of the sensor is the same as that of the optical fibre. 
A variety of methods can be used for interrogating FBG sensors. These include (a) interferometric wavelength shift detection ${ }^{[9,10]}$, (b) Fabry-Perot wavelength filter detection ${ }^{[11]}$, (c) acousto-optic tuning filter techniques ${ }^{[12]}$, (d) edge filter ratiometric method ${ }^{[13]}$ and (e) conventional polychromator. Interferometric methods offer the highest resolution and are suitable for high precision strain measurements. The ratiometric technique offers technical simplicity and fast signal recovery. With respect to dynamic fatigue testing of composites, the cyclic frequency which is typically used is in the range between $2-10 \mathrm{~Hz}$ with a strain range of $-1 \%$ and $+1 \%$. The techniques mentioned above have only been demonstrated for use in the laboratory, further development is needed before they can be used for in-situ strain monitoring during fatigue testing. Interrogation of the FBG using a polychromator is not suitable for in-situ strain measurements in composites due to the slow scanning speed of the instrumentation. Furthermore, the bulkiness of the instrumentation also makes it unsuitable for onsite applications.

In this work, a fibre-optic CCD spectrometer (Ocean Optics Model S1000) was used. This instrument operates in the spectral range 500-1000 nm with a resolution of $0.42 \mathrm{~nm}$ per pixel and response time of $8 \mathrm{~ms}$ per spectrum. The Bragg grating wavelength shift with strain is fairly small, therefore, it was necessary to improve the spectral resolution. Since the FBG sensor reflective spectrum was clearly defined, the application of a spline fitting routine to the spectrum is justified. This curve fitting procedure enabled the spectral resolution to be improved to better than $0.05 \mathrm{~nm}$. This corresponds to a strain sensitivity of approximately $80 \mu \varepsilon$, which fulfils the requirement of the fatigue test undertaken in this study. This CCD-based approach offers access to the whole FBG reflection spectrum rather than just using the peak reflection wavelength. The FBG reflection spectrum is known to become complicated at high strain, hence the peak wavelength may not be related to the actual strain.

\section{PRINCIPLES}

The FBG sensors were made from a standard single-mode fibre which was sensitised by exposing it to a high pressure hydrogen atmosphere. The nominal Bragg grating wavelength was $827 \mathrm{~nm}$ with a bandwidth of 0.2 $\mathrm{nm}$ and a reflectivity of $90 \%$. The Bragg grating wavelength is given by:

$$
\lambda_{b}=2 n \Lambda
$$

where $\mathrm{n}$ is the refractive index of the fibre core, $\Lambda$ is the period of the gratings. When the fibre is subject to a longitudinal strain, the Bragg wavelength will alter due to stress induced change in the spacing of the grating and an associated photoelastic induced change in the refractive index. The fractional change of the Bragg wavelength can be described by ${ }^{[14]}$ :

$$
\Delta \lambda_{b} / \lambda_{b}=\left\{1-\left[\left(n^{2} / 2\right)\left[P_{12}-\mu\left(P_{11}-P_{12}\right)\right]\right\} \varepsilon\right.
$$

where, $\mu$ is the Poisson's ratio, $\mathrm{P}_{12}$ and $\mathrm{P}_{11}$ are the components of the strain-optic tensor of the fibre. Using the values $1.45,0.17,0.121$ and 0.27 for $n, \mu, P_{11}$ and $P_{12}$ respectively, the predicted wavelength shift for the Bragg grating with a central wavelength of $827 \mathrm{~nm}$ is $\sim 0.65 \mathrm{~nm} / 1000 \mu \varepsilon$. These types of grating sensors have been characterised by some of the authors using precision interferometric methods and the wavelength shift coefficient with strain was found to be approximately $0.64 \mathrm{~nm} / 1000 \mu \varepsilon^{[9]}$.

\section{EXPERIMENTAL}

\subsection{Experimental set-up}

The accuracy of the fibre-optic CCD spectrometer was found to be sensitive to the position of the fibre-tip which was housed in the SMA connector, see the insert in Figure 1. A schematic illustration of the experimental set-up which was used for the calibration of the CCD spectrometer and the characterisation of the SLD light source is shown in Figure 1. With reference to Figure 1, a laser light and a broad-band super luminescent diode (SLD) were used to illuminate the single mode couplers. The reflected light from the coupler 
was detected using the CCD spectrometer. The ends of fibre (A) from coupler number 1 and the Bragg grating were immersed in an index matching gel to eliminate reflected light from the end-faces. The nominal central wavelength of the SLD was $840 \mathrm{~nm}$ with a bandwidth of approximately $10 \mathrm{~nm}$. The output power coupled into the single-mode fibre was approximately $100 \mu \mathrm{W}$.

\subsection{Calibration of the CCD spectrometer}

A simple spectral calibration of the CCD spectrometer was carried out using the He-Ne laser, as shown in Figure 1. The reflected light was detected by the CCD spectrometer which in turn permitted the wavelength offset to be calculated. An assumption was made that the offset in the Bragg wavelength at $827 \mathrm{~nm}$ was similar to the offset observed with the He-Ne wavelength at $632.8 \mathrm{~nm}$.

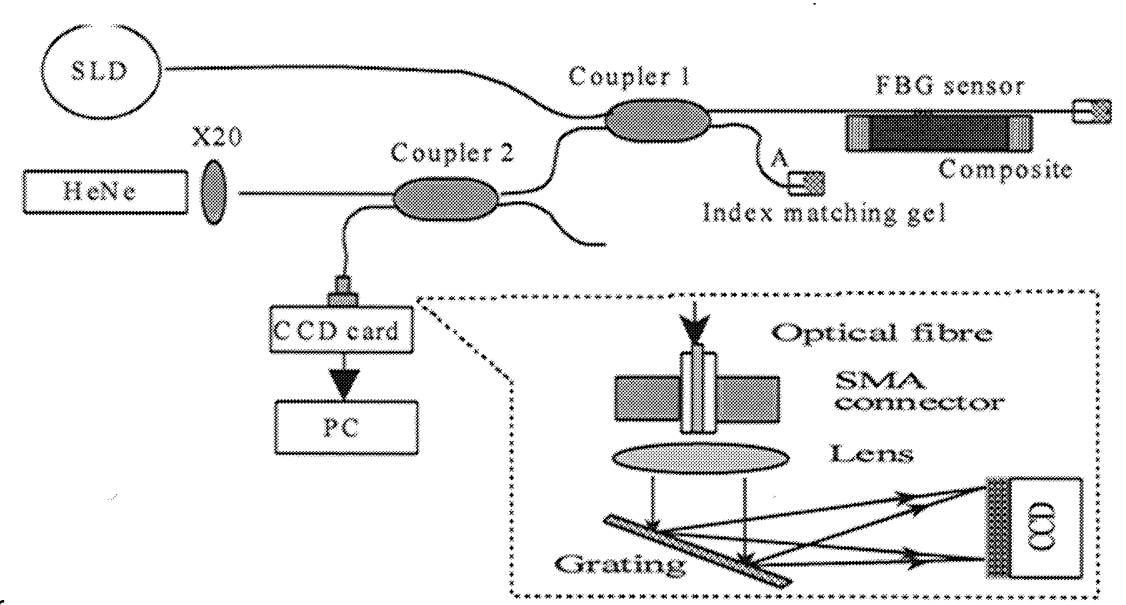

Figure 1 Scnematic illustration of the experimental set-up for the FBG strain measurement experiments.

\section{$\underline{3.3 \text { Fabrication of test specimens }}$}

The prepreg system, T300/920 was purchased from Ciba-Geigy and a 16 ply $\left(0,90_{2}, 0_{2}, 90,0,90\right)_{\mathrm{S}}$ configuration was used. A single specimen mould $(200 \mathrm{~mm} * 20 \mathrm{~mm} * 2.5 \mathrm{~mm})$ was used to fabricate individual test specimens. The prepregs were cured using the manufacturers recommended cure schedule. The leading and trailing ends of the sensor fibre were protected with a polymer tubing. The cured specimens were end-tabbed with aluminium to protect the composite from damage induced by the clamping device during mechanical loading. The FBG sensor was surfaced mounted on to the composite specimen using an adhesive. Prior to this, the bonding-area was first lightly abraded and degreased using conventional procedures.

\subsection{Tensile and compressive loading}

The preliminary static tensile tests were carried out on an Instron 4206 testing machine. All subsequent tensile and compressive tests were conducted using an Instron 8501 servo-hydraulic dynamic testing machine. An anti-buckling jig was used to carry out the compressive loading experiments. The fatigue tests were carried out using a range of loading frequencies from $0.1 \mathrm{~Hz}$ to a maximum of $6 \mathrm{~Hz}$. A stress ratio of -0.5 was used for all the fatigue experiments.

\section{RESULTS AND DISCUSSION}

\subsection{Calibration of the CCD}

The reflection spectrum obtained from the CCD spectrometer is shown in Figure 2. The CCD reading from the He-Ne laser was $630.52 \mathrm{~nm}$, thus indicating an offset of approximately $-2.3 \mathrm{~nm}$. The SLD central wavelength was about $20 \mathrm{~nm}$ longer than its nominal output wavelength of $840 \mathrm{~nm}$. In Figure 2, the residual 
reflection from the optical fibre-ends is larger than the FBG reflection, this is because the Bragg grating wavelength was located at the edge of the SLD emission spectrum.

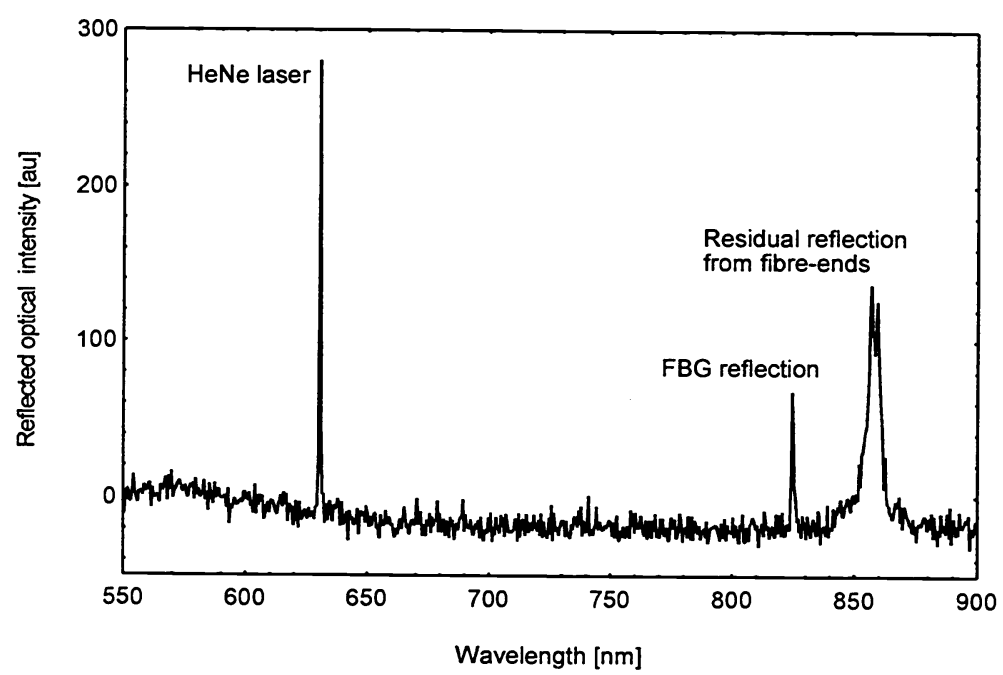

Figure 2 The reflection spectrum of the FBG using a He-Ne laser source.

Figure 3 illustrates the reflected Bragg grating wavelength under tension-free conditions. The spectrum indicates a peak centred at approximately $824.6 \mathrm{~nm}$, however, if a correction is applied to take into account the offset in the CCD spectrometer, then the value of $826.8 \mathrm{~nm}$ is close to the nominal wavelength of $827 \mathrm{~nm}$.

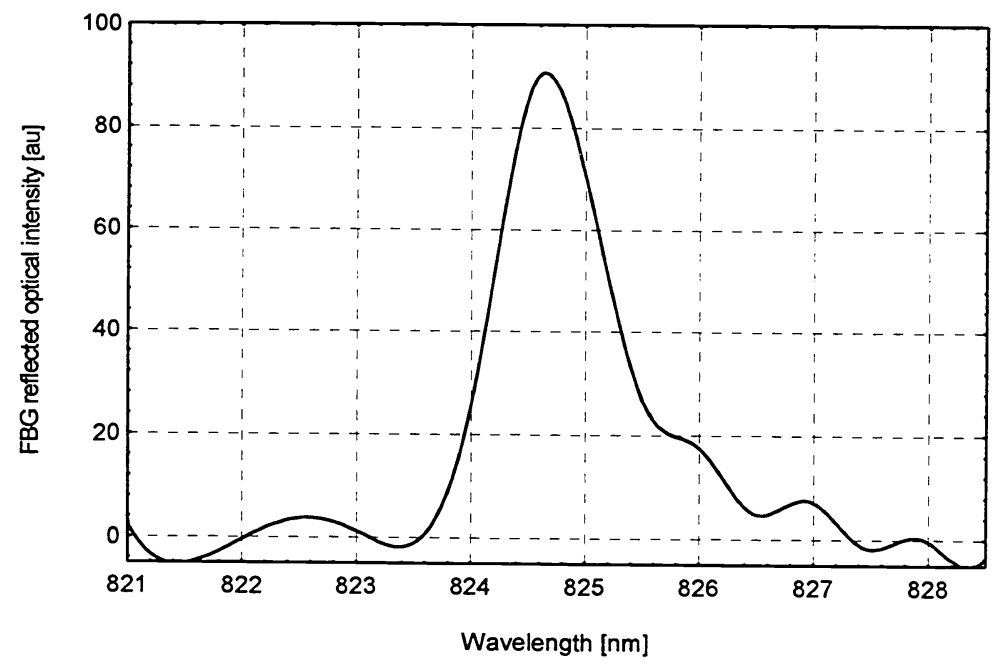

Figure 3 An example of a typical reflection spectrum from the FBG sensor.

\subsection{Static test results}

Figure 4 illustrates the response of the surface mounted FBG sensor to a series of quasi-static strains. The computed relationship between applied strain and the Bragg reflection is $0.65 \mathrm{~nm}$ per milli-strain. This correlates with the results obtained using the extensometer for both tensile and compressive loading, see Figure 5. The strain range for these experiments were restricted to between $-0.2 \%$ and $0.3 \%$ primarily due to the limited number of FBG sensors which were available for this study. 


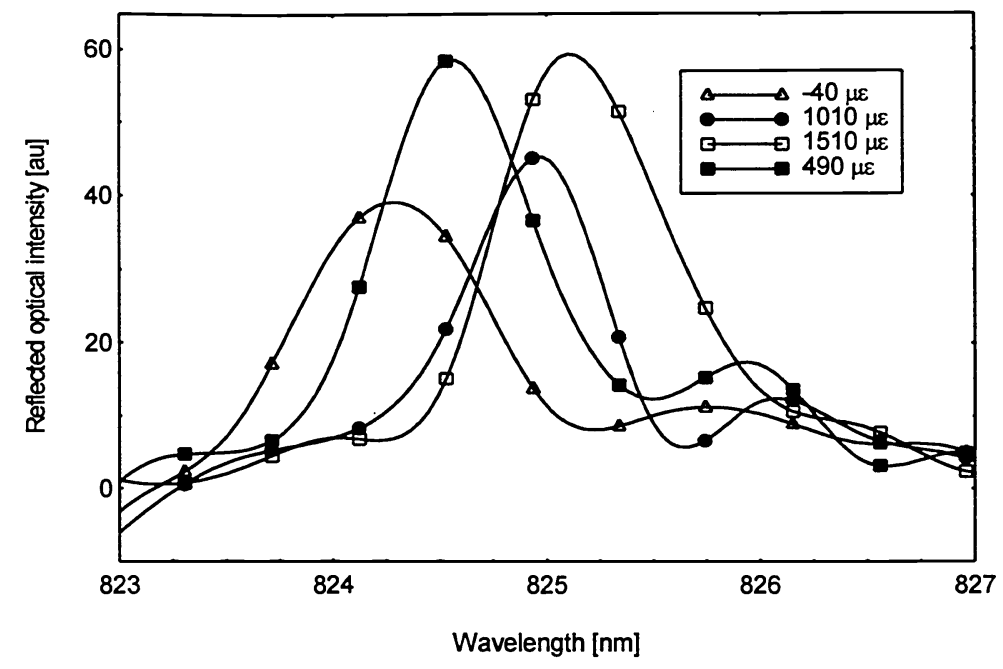

Figure 4 Typical reflection spectra from the FBG as a function of various applied tensile strains.

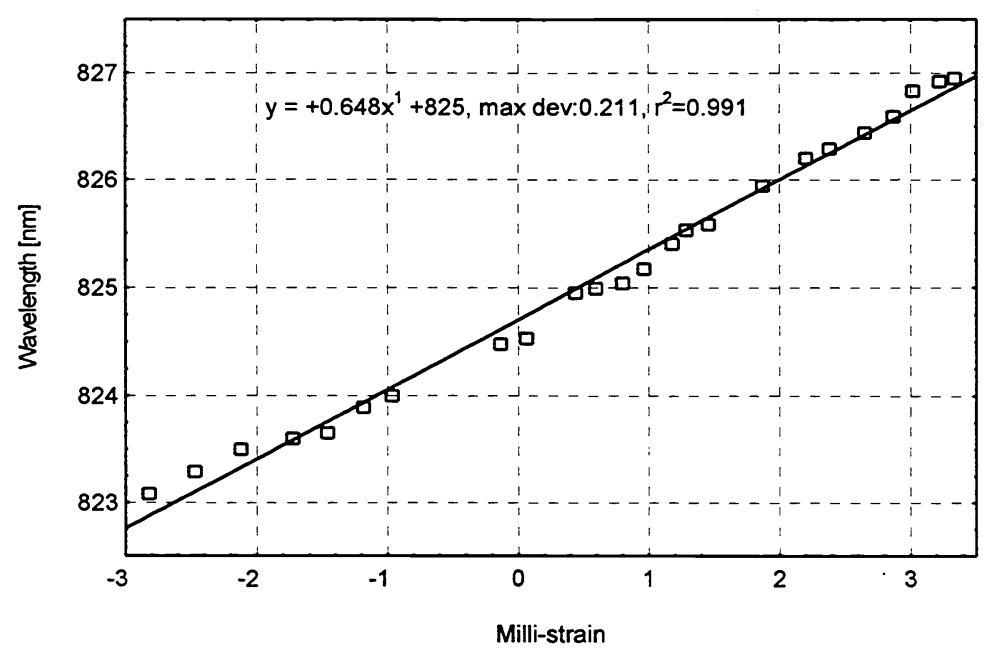

Figure 5 Optical wavelength versus milli-strain for the surface mounted FBG sensor.

\subsection{Fatigue test results}

The primary aims of the fatigue study were three-fold. Firstly, it was necessary to establish the durability of the FBG under dynamic loading conditions. Secondly, to establish the correlation between the conventional method for measuring the dynamic stiffness using an extensometer and the output from the FBG sensor. Thirdly, to demonstrate that the FBG sensor system used in this study was capable of detecting the stiffness change in the composite as a function of cyclic loading. The influence of temperature was not considered in this study because the stress/strain data presented in Figures 6 and 7 were generated at a constant temperature of $23^{\circ} \mathrm{C}$.

Figure 6 illustrates excellent correlation between the strain data obtained via the extensometer and the FBG sensor. The fatigue tests were interrupted at regular intervals to obtain stress/strain data for the composite. A very small but progressive deviation of the strain data was observed between the extensometer and the FBG sensor as a function of fatigue cycles. This discrepancy between the two sets of strain measurements is apparent in Figure 7, which presents the stress/strain data obtained after 266,000 cycles. The most probable cause for 
this is the debonding of the surface mounted FBG sensor. The regression equation presented in Figures 6 and 7 correspond to the data from the FBG.

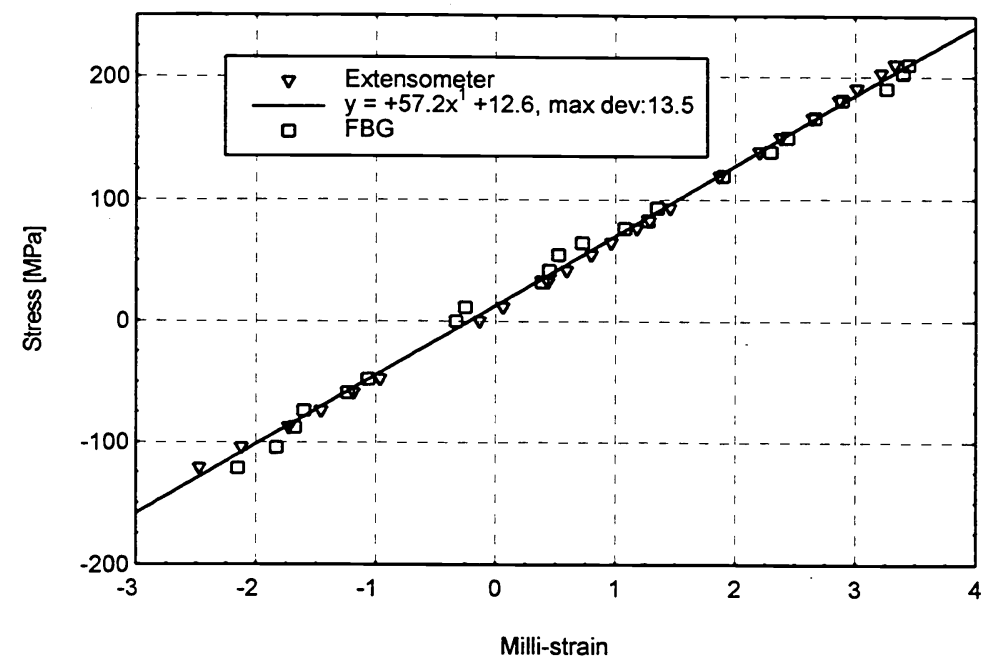

Figure 6 Stress/strain curve after 300 cycles obtained with an extensometer and a FBG sensor.

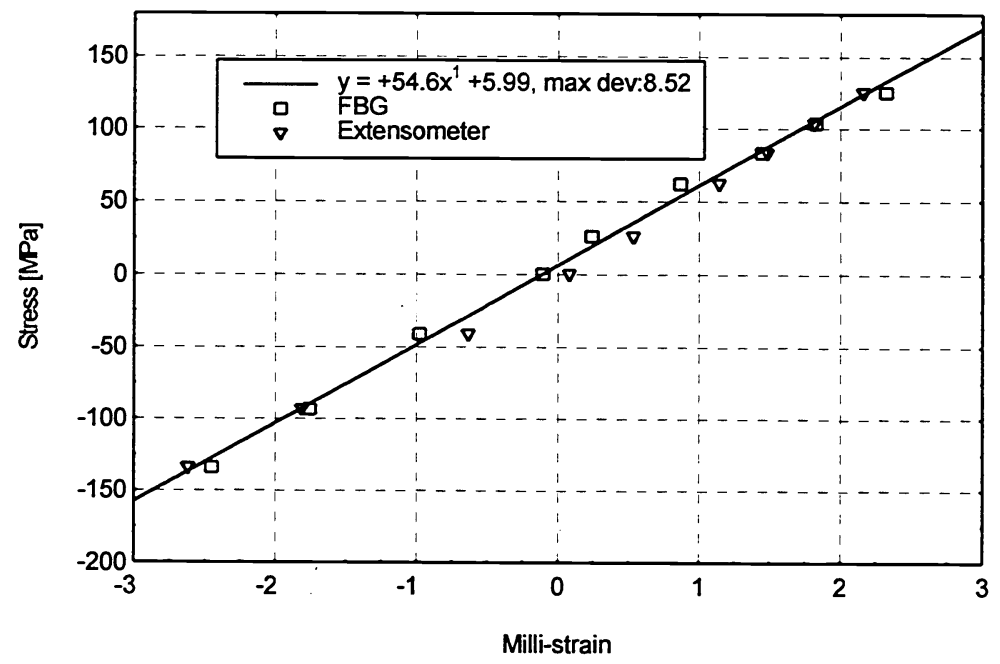

Figure 7 Stress/strain curve after 266,000 cycles obtained with a surface mounted extensometer and a surface mounted FBG sensor.

Further evidence for this debonding problem can be seen on comparing the FBG reflection spectra after 300 and 536,000 cycles as shown in Figures 8 and 9 respectively. A distinct broadening of the spectrum can be seen as a function of fatigue cycles. As a consequence of this observed broadening of the Bragg spectrum, the FBG sensor was removed and then secured back on the surface of the composite until the termination of the fatigue test at 700,000 cycles. The details of a study on this spectrum broadening effect and the use of embedded FBG sensors for in-situ stiffness measurements will be reported in a subsequent publication. Preliminary results indicate that the observed broadening of the Bragg reflection may be used to study the durability of adhesive joints and delaminations in composites. 


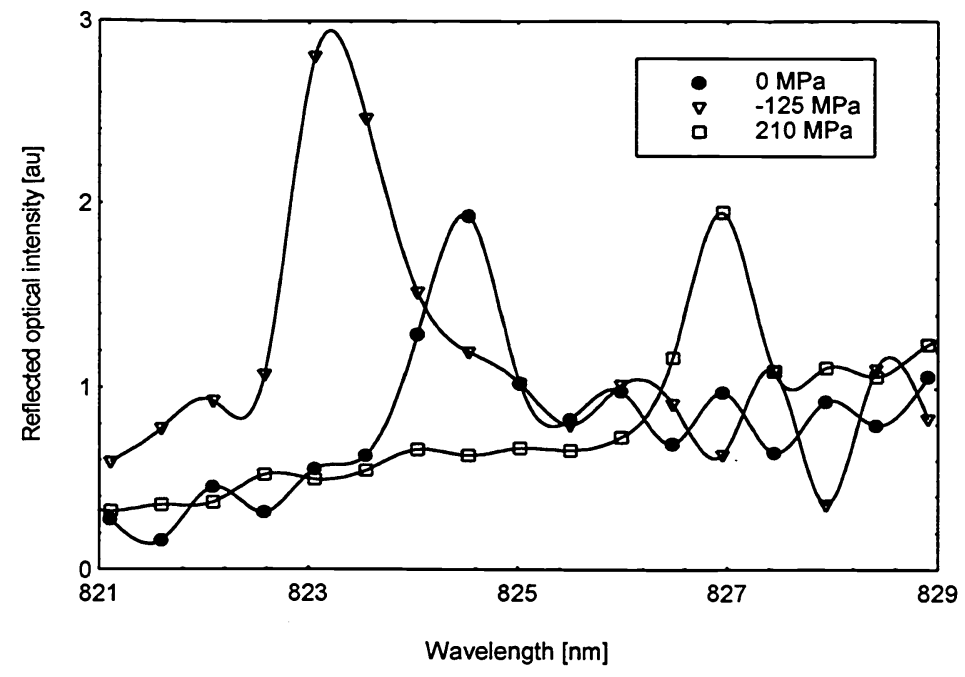

Figure 8 FBG reflection spectra at $0,-125$ and $210 \mathrm{MPa}$, obtained after 300 cycles.

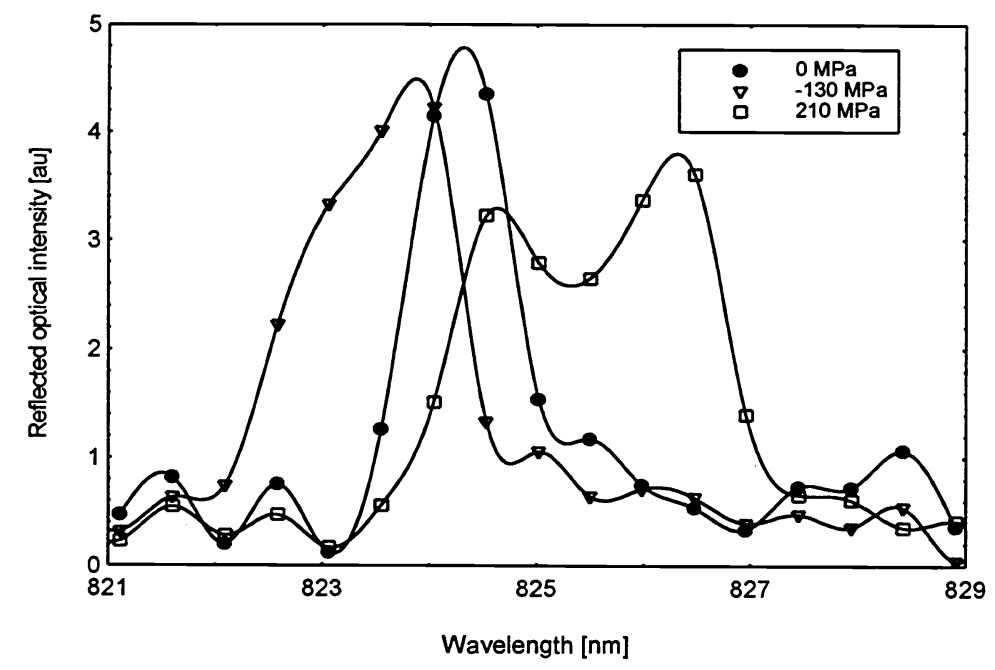

Figure 9 FBG reflection spectra at $0,-130$ and $210 \mathrm{MPa}$, obtained after 536,000 cycles.

Figure 10 demonstrates the stiffness reduction in the composite as a function of fatigue cycling using the fatigue test conditions mentioned in the previous section. Both the extensometer and the FBG sensors showed a similar trend with respect to stiffness reduction. Figure 10 clearly demonstrates that the FBG sensors can be used to monitor the stiffness reduction in composites under dynamic loading conditions. The discrepancy between extensometer and FBG data may be attributed to debonding of the FBG from the composite. It is envisaged that surface treating the FBG with a suitable coupling agent will enhance the bond strength. Embedding the FBG sensor may also overcome this problem. 


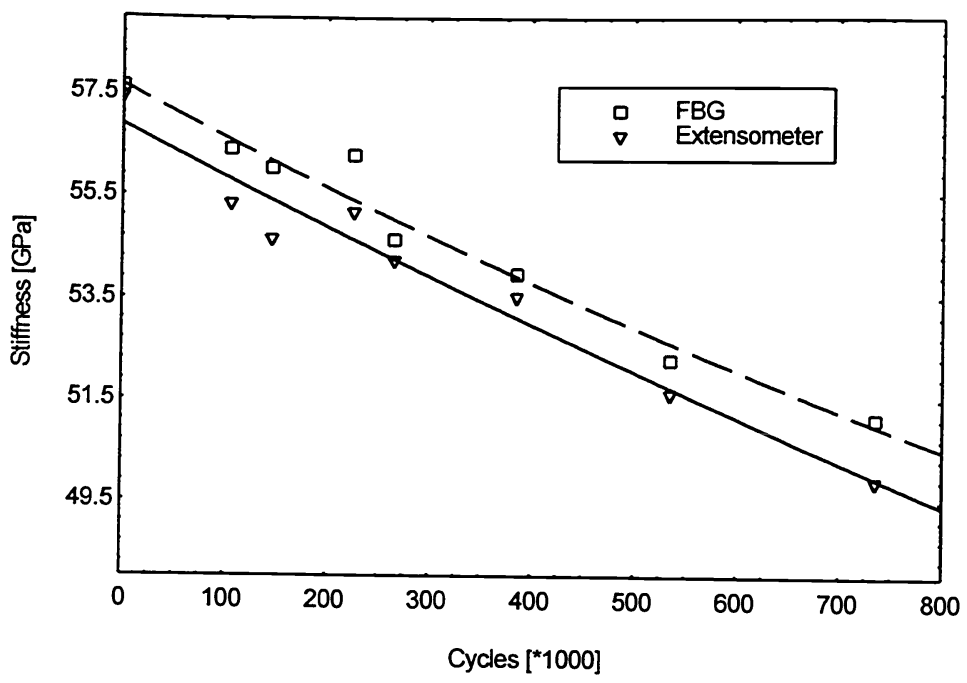

Figure 10 Stiffness-decay data using a surface mounted extensometer and a FBG sensor. The composite was under fatigue testing using a peak stress of $210 \mathrm{MPa}$, a frequency of $6 \mathrm{~Hz}$ and a stress ratio of -0.5 .

\section{SUMMARY}

This study has successfully demonstrated the use of a relatively low resolution CCD spectrometer for conducting strain measurements in composite test specimens using an in-fibre Bragg grating sensor. The sensor system used in this study was also capable of measuring the relative reduction in the stiffness of the composite as a function of fatigue cycles. The sensitivity of static strain measurements was approximately 80 $\mu \varepsilon$ and the system has a response time of less than $8 \mathrm{~ms}$. This preliminary study has demonstrated that the FBG sensor system used in this study survived cyclic tensile and compressive fatigue loading up to the termination of the fatigue test at 700,000 cycles. Debonding of the FBG sensor from the surface of the composite was identified as a potential problem for long term strain monitoring using surface mounted sensors. A broadening of the reflected Bragg spectra was observed for a range of applied strains and this broadening led to a small but noticeable discrepancy in the measured strain data between the surface mounted extensometer and the FBG sensor.

Future work will be carried out to facilitate real-time strain monitoring during fatigue testing. In the current work, the fatigue tests were interrupted to enable stress/strain data to be collected. Compared to other signal processing methods, the CCD spectrometer offers the advantage of acquiring information on the characteristics of the reflection spectra throughout the whole lifetime of the test.

\section{ACKNOWLEDGEMENT}

T.Liu acknowledges the EPSRC for the award of a ROPA grant. The authors also wish to acknowledge the useful discussions with S. Kanellopoulos of King's College London and the assistance given by K. Hale and C. France of the Department of Manufacturing and Engineering Systems, Brunel University.

\section{REFERENCES}

1. T. K. O'Brien and K. L. Reifsinder: "Fatigue damage evaluation through stiffness measurements in boronepoxy laminates", Journal of Composite Materials Vol. 15, pp.55-70, 1981. 
2. R. M. Measures, "The detection of damage and the measurement of strain within composites by means of embedded optical fibre sensors", Review of Progress in Quantitative Non-Destructive Evaluation, Vol. 10 B, pp.1247-1257, 1991.

3. H. Hogg, B. Mason, T. Valis and R. M. Measures, "Development of a fibre Fabry-Perot (FFP) strain gauge system", Active Materials and Adaptive Structures, G. J. Knowles editor, pp. 667-672, 1992, IOP Publishing, Bristol and Philadelphia.

4. R. O. Claus, M. S. Gunther, A. B. Wang, K. A. Murphy and D. Sun, "Extrinsic Fabry-Perot sensors for structural evaluation", Applications of fibre optic sensors in engineering mechanics, Farahad Ansari, Editor, pp.
$60-70,1993$.

5. R. Badcock and G. F. Fernando, "An intensity based optical fibre sensor for fatigue damage detection in advanced fibre-reinforced composites", Smart Mater. and Struct., Vol. 4, pp. 223-230, 1995.

6. B. E. Jones and R. A. Spoocer, "Photoelastic pressure sensor with optical fibre links using wavelength characterisation", Proc. First International Conference on Optical Fibre Sensors, pp. 173, London, 1983.

7. K. O. Hill, "Fibre Bragg gratings: properties and sensing applications", Conference Proceedings OFS-11, pp. 92-94. May, 1996, Hokkaido, Japan

8. H. D. Simonsen, and R. Paetsch, "Fibre Bragg grating sensor demonstration in a glass-fibre reinforced polyester composite", 1st European Conference on Smart Structures and Materials, Glasgow, pp. 73-76, 1992.

9. Y. J. Rao, A. B. Lobo Ribeiro, D. A. Jackson , L. Zhang and I. Bennion, "Combined spatial- and timedivision-multiplexing scheme for fibre grating sensors with drift-compensated phase-sensitive detection", Optics Letters, Vol. 20, No. 20, pp.2149-2151, 1995.

10. A. D. Kersey, T. A. Berkoff, and W. W. Morey, "High resolution fibre-grating based strain sensor with interferometric wavelength-shift detection”, Electron. Lett., Vol. 28, No. 3, pp. 236-238, 1992.

11. A. D. Kersey, T. A. Berkoff, and W. W. Morey, "Multiplexed fibre Bragg grating strain-sensor system with a fibre Fabry-Perot wavelength filter", Optics Letters, Vol. 18, No. 16, pp. 1370-1372, 1993.

12. M. G. Xu, H. Geiger, J. L. Archambault, L. Reekie, and J. P. Dakin, "Novel interrogation system for fibre Bragg grating sensors using an acousto-optic tunable filter", Electron. Lett., Vol. 29, No. 17, pp. 1510-1511,
1993.

13. R. M. Measures, S. Melle and K. Liu, "Wavelength demodulation Bragg grating fibre optic sensing systems for addressing smart structure critical issues", Smart Mater. and Struct., Vol. 1, pp. 36-44, 1992.

14. W. W. Morey, G. Meltz, and W. H. Glenn, "Fibre optic Bragg grating sensors", SPIE Vol. 1169, Fiber Optic and Laser Sensors VII pp. 98-107, 1989. 